\title{
LA EDUCACIÓN PARA LA CIUDADANÍA DEMOCRÁTICA EN EUROPA
}

\author{
EDUCAÇÃO PARA A CIDADANIA DEMOCRÁTICA NA EUROPA
}

\section{Mariona Massip Sabater ${ }^{1}$ \\ Antoni Santisteban Fernández ${ }^{2}$}

\begin{abstract}
Resumen: En los diferentes países europeos se presentan distintas maneras de proyectar la Educación para la Ciudadanía. Aun así, se desarrollan sobre unas bases compartidas: la defensa de los derechos humanos o la educación para la paz, entre otras. Estas diferencias están marcadas por las distintas tradiciones en cultura o en la historia democrática de los diferentes estados, así como por las diferentes perspectivas ideológicas con las que se entiende la democracia y la educación. El Consejo de Europa promueve algunas directrices generales para poder incorporar en los diferentes currículos de los países la Educación para la Ciudadanía, en la actualidad, a partir de una propuesta de Competencias de Cultura Democrática. Algunos estudios revelan que la Educación para la Ciudadanía está presente en los distintos currículos, así como la escala europea y global. En todos los países la formación del profesorado se considera la clave para poder implementar los programas con éxito, para hacer frente a los retos actuales, como son el aumento de los discursos del odio y discriminatorios, la disminución de la participación ciudadana o la convivencia intercultural.
\end{abstract}

Palabras clave: Educación para la Ciudadanía, Consejo de Europa, Competencias de Cultura Democrática, Europa, Participación Ciudadana.

\begin{abstract}
Different ways of projecting Citizenship Education are presented in different European countries. Even so, they share some cornerstones: the defense of human rights or peace education, among others. These differences are caused by different traditions in culture and culture history, and by the different ideological perspectives to understand Democracy and Education. Council of Europe promotes some general guidelines to establish Citizenship Education, currently, based on a proposal Competences for Democratic Culture. Some studies reveal that Citizenship Education is present in the different curricula, as well as the European and global scales. Teacher training is understood as the key to the successful implementation of these programs, with the objective of addressing the challenges of the current context: the increase in hate and discriminatory discourses, the decrease in citizen participation and intercultural coexistence.
\end{abstract}

Key words: Citizenship Education, Council of Europe, Competences for Democratic Culture, Citizen Participation.

Resumo: Diferentes formas de projetar a Educação para a Cidadania são apresentadas em diferentes países europeus. Mesmo assim, eles se desenvolvem em bases compartilhadas: a defesa dos direitos humanos ou a educação para a paz, entre outros. Essas diferenças são marcadas pelas distintas tradições culturais ou históricas democráticas dos diferentes estados, bem como pelas diferentes perspectivas ideológicas com as quais a democracia e a educação são entendidas. O Conselho da Europa promove algumas diretrizes gerais para incorporar a Educação para a Cidadania nos diferentes currículos dos países atualmente, com base em uma proposta de Competências Culturais Democráticas. Alguns estudos revelam que a Educação para a Cidadania está presente nos diferentes currículos, bem como nas escalas européia e global. Em todos os países, a formação de professores é considerada a chave para a implementação bem-sucedida de programas, para enfrentar os desafios atuais, como aumento do ódio e discursos discriminatórios, diminuição da participação do cidadão ou coexistência intercultural.

Palavras-chave: Educação para a Cidadania, Conselho da Europa, Competências para a Cultura Democrática, Europa, Participação Cidadã.

1 Doctoranda de Didáctica de las Ciencias Sociales en la Universitat Autònoma de Barcelona (UAB). Miembro de GREDICS (2017SGR1600). E-mail: mariona.massip@uab.cat. ORCID: https://orcid.org/0000-0002-7054-8819. 2 Doctor en Didáctica de las Ciencias Sociales en la Universitat Autònoma de Barcelona (UAB), profesor de Didáctica de las Ciencias Sociales y director del Departamento de Didáctica de la Lengua, la Literatura y las Ciencias Sociales en la Universitat Autònoma de Barcelona (UAB). Coordinador de GREDICS (2017SGR1600). E-mail: antoni.aantisteban@uab.cat. ORCID: https://orcid.org/0000-0001-7978-5186. 


\section{INTRODUCCIÓN}

En los últimos años en Europa se está viviendo un aumento de discursos populistas, de grupos de ultraderecha y de acciones fascistas, con representación no sólo en espacios públicos y digitales, sino también en espacios de representación política e institucional. Esta realidad se da en un contexto de globalización y de asimilación de retos planetarios (SANT; DAVIES; PASHBY; SHULTZ, 2018), en los que se prevé el incremento de desplazamientos, migraciones y personas refugiadas. Decidir si este mundo global será una casa de acogida para todos y todas, o una tierra de disputa depende de las decisiones que tomemos y de la realidad que construyamos en el presente y en el futuro próximo (Garcés, 2018).

La juventud no sólo vive en esta realidad, sino que es agente activo en su construcción. No sólo es receptora de discursos discriminatorios, sino agente político en su deconstrucción (RANIERI, 2016). No sólo es víctima de la emergencia climática y sus consecuencias, sino que es activista y participa en la lucha contra sus efectos. En este sentido, la Educación para la Ciudadanía es un factor fundamental para preparar a la juventud para que ejerza sus derechos democráticos y se enfrente a los retos fundamentales que tiene por delante.

En este artículo se plantea una revisión de la situación y los retos de la Educación para la Ciudadanía en Europa. En un primer apartado analizamos el concepto de "Educación para la Ciudadanía Democrática" y de "Europa", desde su complejidad y dimensión plural (2. Concreción Conceptual: Educación para la Ciudadanía Democrática y Europa). En segundo término, la diversidad de contextos y el papel del Consejo de Europa en el diseño de directrices comunes y el desarrollo de la propuesta de las competencias de cultura democrática, partiendo de las dinámicas distintas de los países europeos (3. Diferencias y Directrices: El Consejo de Europa). Seguidamente, se repasan algunas de las investigaciones más significativas en los diferentes países europeos, con especial atención a las que refieren a la formación del profesorado (4. La investigación sobre el estado de la Educación para la Ciudadanía en Europa). Finalmente, planteamos algunos retos de futuro (5. Retos y conclusiones).

\section{CONCRECIÓN CONCEPTUAL: EDUCACIÓN PARA LA CIUDADANÍA DEMOCRÁTICA Y EUROPA}

Una revisión de la Educación para la Ciudadanía Democrática en Europa requiere, en primer lugar, una breve reflexión sobre a qué nos referimos cuando hablamos de Educación para la Ciudadanía y de Democracia, y a qué nos referimos cuando hablamos de Europa. Ambos conceptos pueden entenderse desde diferentes perspectivas ideológicas y políticas, siendo conceptos complejos y dinámicos.

Sant (2019) detecta diferentes significados atribuidos a la Educación Democrática desde sus tradiciones y tendencias. Este concepto se considera un punto nodal (LACLAU; MOUFFE, 2001, apud SANT, 2019), de discursos no sólo diferentes, sino muchas veces opuestos y contradictorios. Se considera como un concepto con significado flotante (LACLAU, 2005), un campo de debate donde, desde diferentes posiciones ideológicas, se intenta imponer significado. En una investigación reciente Sant (2019) detecta 7 discursos diferentes subyacentes a la Educación Democrática en publicaciones de la última década.

1) Elitista. Se defiende una educación diferenciada en función del rol social de las personas y colectivos, entendiendo que las élites culturales e intelectuales ejercen de líderes positivos y naturales de la sociedad.

2) Liberal. Se estructura sobre la idea de la libertad como valor más importante. Desde esta perspectiva, la igualdad política podrá ser asumida con la educación de ciudadanos racionales y responsables que conozcan las instituciones, los valores democráticos y los derechos, y piensen de manera crítica. Según esta revisión: "cosmopolitan liberals advocate the need for a cosmopolitan democratic education that examines the ethical basis of human rights" (p.664).

3) Neoliberal. Desde esta perspectiva, la democracia se convierte en el equival ente político de la economía de mercado e incorpora ideas agresivas sobre la competición entre individuos: "Citizens are conceived as rational consumers who, through voting, compete so that their views and private interests prevail" (p.665). En este caso, los mercados son entendidos como un buen organizador social y se espera que las personas, movidas por el propio interés, mejoren la realidad social y económica. La educación se cuantifica a través de estándares y se aboga a la sustitución de la escuela pública por diferentes opciones 
privadas en las que familias y alumnado son entendidos como consumidores.

4) Deliberativa. Se defiende un espacio público en el que toda la ciudadanía pueda exponer y debatir en condiciones de igualdad. Se da importancia a la comunicación, al razonamiento y a la inclusión, y se considera a toda la ciudadanía como coautora de las decisiones públicas. Los debates en educación se centran en quién toma las decisiones educativas y cómo deberían tomarse, así como en la importancia de trabajar con temas controvertidos, la educación mediática, el arte, la filosofía y las ciencias sociales.

5) Multicultural. Se da prioridad a los debates sobre pluralidad y diversidad, entendiendo que la libertad y el respeto por la diferencia son dos pilares democráticos. Se defiende la pluralidad de maneras de ser y el particularismo, renunciando a visiones más universalistas de las personas y la ciudadanía. Se aboga por un currículum que relacione poder y cultura, y que cuestione el eurocentrismo.

6) Participativa. Se defiende la participación como un elemento clave de la democracia. La ciudadanía se entiende en el marco de la vida asociativa, en la organización de los individuos en colectivos que actúan con libertad. Desde esta perspectiva, la educación tiene que centrarse en la acción y en la intervención social, y el centro educativo tendría que ser un espacio en el que poder participar.

7) Crítica. Esta perspectiva se articula sobre las ideas de la igualdad y la transformación social. Defiende una educación que garantice que todos los seres humanos tengamos oportunidades equitativas, como motor de auténtica transformación social, denunciando aquellos mecanismos de poder y opresión que generan desigualdad. En este sentido, la educación es entendida como la herramienta para revertir las injusticias sociales a través de la emancipación.

La gestión de la pluralidad y la diversidad, de discursos y prácticas procedentes de ideologías y paradigmas distintos, supone un reto en la construcción de un marco común. A esta pluralidad se le añade, como veremos, los diferentes enfoques de la Educación Democrática o la Educación para la Ciudadanía Democrática en los distintos países europeos, que tienen también recorridos históricos y culturales diferentes en el ejercicio de la democracia. El Consejo de Europa concreta la idea de Democracia de la siguiente manera ${ }^{3}$ :

La Democracia, como se interpreta de manera común, es una forma de gobierno ejercida por o en nombre de la gente. La principal función de esta forma de gobernanza es ser sensible a las visiones de la mayoría. Por esta razón, la democracia no puede operar en ausencia de las instituciones que aseguran el derecho a voto de los ciudadanos adultos, la organización de elecciones libres, justas y regulares, las leyes de la mayoría y la responsabilidad del gobierno. Aun así, aunque la democracia no puede existir sin las instituciones democráticas y las leyes, estas instituciones no pueden funcionar sin una ciudadanía que practique una cultura democrática y mantenga actitudes y valores democráticos. Entre otros, estos incluyen:

1. El compromiso con los debates y deliberaciones públicos

2. La voluntad de expresar las propias opiniones y escuchar las opiniones de los demás

3. La convicción que la diferencia de opiniones y los conflictos se deben resolver de manera pacífica

4. El compromiso con la protección de las minorías y sus derechos

5. El reconocimiento de que las leyes de la mayoría no pueden abolir los derechos de las minorías

6. El compromiso con las leyes

7. La Democracia también requiere el compromiso de la ciudadanía a participar activamente en la esfera pública. Si los ciudadanos y las ciudadanas no se adhieren a estos valores, prácticas y actitudes, las instituciones democráticas no pueden funcionar. (COUNCIL OF EUROPE, 2018a, pp.23-24)

La idea de Europa también requiere de algunas explicaciones, ya que puede interpretarse como concepto geográfico o político. Desde una perspectiva geográfica, Europa es un continente en el que se

3 Traducido del inglés por los autores del artículo. 
engloban 50 estados reconocidos por la $\mathrm{ONU}^{4}$. Más de la mitad de estos se organizan en la entidad supranacional de la Unión Europea, que a día de hoy cuenta con 27 estados miembros. Europa, como concepto político, se usa en algunos casos como sinónimo de Unión Europea, aunque esta sea una entidad dinámica. Desde sus inicios en 1958 con 6 estados miembros, se han ido incorporando países. El 2013 se llegó a los 28 países miembros. En 2020 Reino Unido ha dejado de formar parte de ella.

Por otro lado, el Consejo de Europa es una organización internacional de la que forman parte la mayor parte de países europeos (47), con objetivo de garantizar los derechos humanos. En este artículo nos referimos, sobretodo, a los esfuerzos, campañas e investigaciones educativas del Consejo de Europa.

\section{DIFERENCIAS Y DIRECTRICES: EL CONSEJO DE EUROPA}

El Consejo de Europa desarrolla, desde 1997, el proyecto Education for Democratic Citizenship (EDC). Se trata de un foro de debate entre expertos y profesionales de todos los estados miembros, para definir los conceptos, estrategias y buenas prácticas. Dado que existe una evidente pluralidad en los enfoques sobre la educación democrática y la educación para la ciudadanía en los diferentes países, ha sido necesario, y sigue siéndolo, un trabajo permanente de debate y consenso (GOMES; GÓMEZ; SANTISTEBAN; PAGĖS, 2020). Esta pluralidad se entiende, por un lado, debido a las distintas tradiciones de los países de la Unión Europea, no sólo sobre educación, sino en cultura y práctica democrática. Existe una marcada diferencia entre aquellos países con una situación política más estable y aquellos que han vivido situaciones recientes de conflictos y tensiones (GALLAGHER; DUFFY, 2016). La incorporación de países de la Europa del Este a la UE ha hecho aún más evidente estas diferentes tradiciones.

Por otro lado, por las diferentes perspectivas ideológicas subyacentes en los discursos sobre Educación Democrática, tratadas anteriormente. Finalmente, cabe destacar otro elemento que favorece la diversidad de planteamientos en los distintos países. Se detecta en los países nórdicos un índice más elevado de equidad y oportunidades educativas, en contraste con otras regiones con mayores desigualdades económicas (HALLDÓRSDÓTTIR; PALL; RÓS, 2016; WILKINSON; PICKET, 2009). Las perspectivas con las que se afrontan temas como la integración, la diversidad, la convivencia, la participación o la igualdad en términos educativos dependen en gran medida del contexto social en los que se desarrollan (HALLDÓRSDÓTTIR; PALL; RÓS, 2016).

La gestión de la pluralidad de ideologías y de la diversidad existente en los distintos países supone un reto para la proyección de unas directrices comunes sobre Educación Democrática en Europa. En cada país se han seguido procesos diferentes. En algunos de ellos, los sectores religiosos, conservadores o ultraconservadores han ejercido una fuerte oposición a estos programas de Educación para la Ciudadanía. El Consejo de Europa, que tiene como uno de sus principales objetivos promover la educación para una ciudadanía activa, inclusiva y orientada a la cohesión social, ha realizado diferentes estudios sobre la situación en cada país, para conocer los avances realizados en cada uno de ellos, visualizar la situación de conjunto y hacer recomendaciones necesarias.

Dichas recomendaciones se concretan en un documento publicado en 2016, "Competences for democratic culture. Living together as equals in culturally diverse democratic societies". En este se incluyen 20 competencias, agrupadas en 4 grandes bloques: valores, actitudes, habilidades y conocimientos (imagen 1). Estas competencias se plantean para dar respuesta a algunos retos que ponen en peligro la legitimidad de las instituciones europeas y la convivencia pacífica en Europa: el descenso de la participación electoral, el aumento de la desconfianza hacia representantes políticos, el aumento de los crímenes y los discursos del odio, la intolerancia y los prejuicios respecto a minorías étnicas y religiosas, y el aumento del extremismo violento (COUNCIL OF EUROPE, 2016). La propuesta enfatiza la importancia de dar valor a la dignidad humana, a los derechos humanos, a respetar y valorar la diversidad. Se entiende el aprendizaje de conocimientos y conceptos sociales para interpretar críticamente del mundo, así como para hacer frente a los retos actuales y futuros.

4 Rúsia, Georgia y Azerbaidjan tienen parte de sus territorios en la Europa geográfica, por lo que se consideran países europeos. Por otro lado, Chipre y Armenia tendrían que ser considerados países asiáticos por su situación geográfica, pero se consideran europeos por cuestiones históricas y culturales.

Rev. Espaço do Currículo (online), João Pessoa, v.13, n.2, p. 142-152, maio/agos. 2020. 
Imagen 1 - Competencias para la cultura democrática.

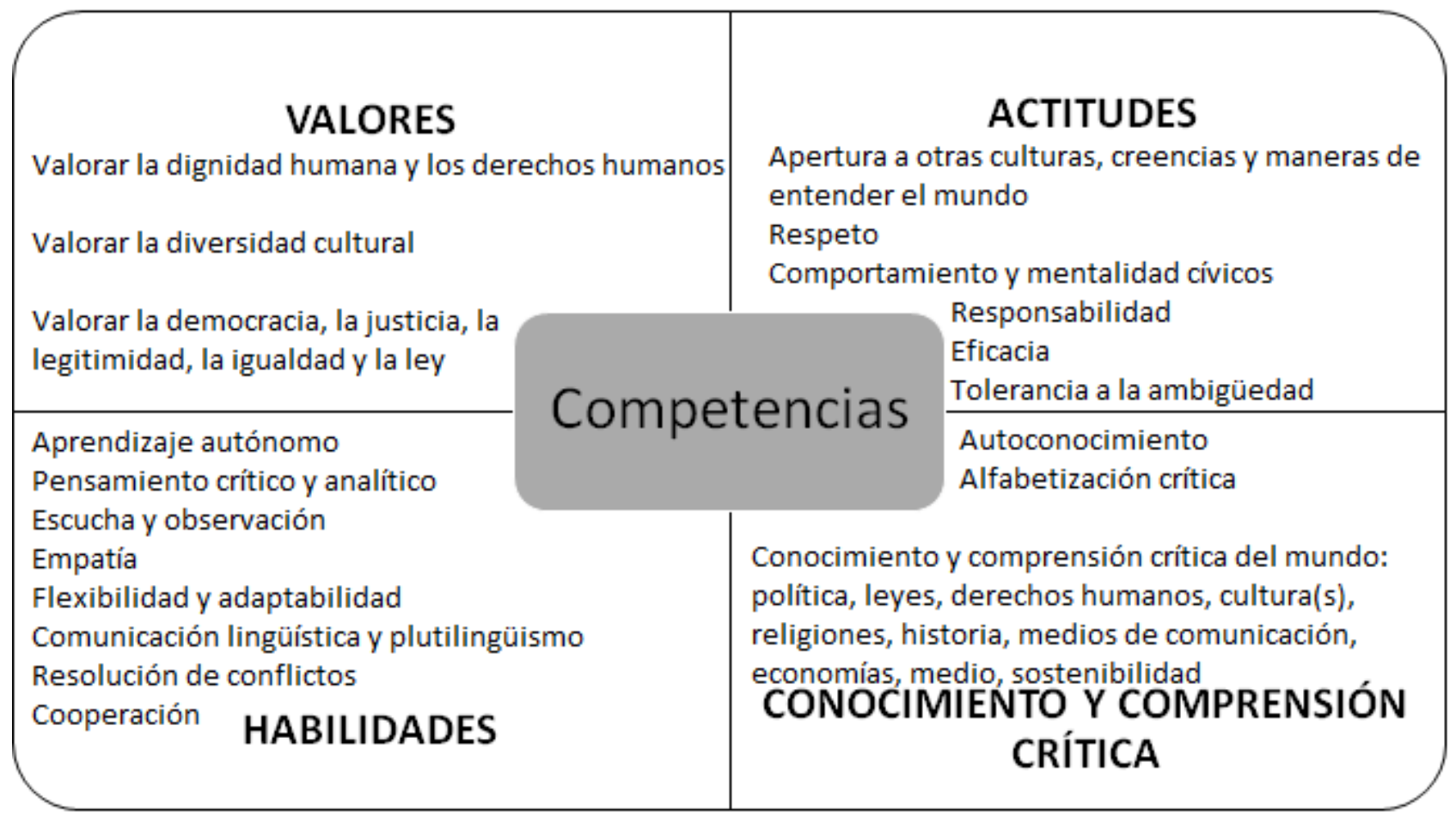

Fonte: Consejo de Europa (2016)

En 2018 se publica un documento más extenso, "Reference Framework of Competences for Democratic Culture", que consta de 3 volúmenes: Contexto, Conceptos y Modelo (V.1); Descriptores de Competencias para la Cultura Democrática (V.2), y Guía para la Implementación (V.3). El documento parte del modelo competencial de 2016 y lo desarrolla de manera amplia. En este, se entienden las competencias como la "habilidad de movilizar y desplegar valores, actitudes, habilidades y conocimientos relevantes para dar respuesta de manera apropiada y efectiva a las demandas, retos y oportunidades de cada contexto" (COUNCIL OF EUROPE, 2018a, p.32). Desarrolla, enmarca y ubica conceptos como identidad(es), cultura, intercultural y diálogo intercultural (2018a); de hecho, señala el diálogo intercultural como uno de les ejes vertebradores de la cultura democrática. Se entiende que "en sociedades culturalmente diversas, las instituciones y procesos democráticos requieren diálogo intercultural", para "asegurar que todos los ciudadanos y ciudadanas tengan igualdad de posibilidades a la hora de participar en las discusiones públicas y la toma de decisiones" (2018a, p.24). A su vez, dicho diálogo intercultural requiere respeto para cada uno de los interlocutores y garantizar que la comunicación no sea contradictoria o coercitiva, ya que entonces no sería un diálogo, sino intentos de demostrar la superioridad de unos argumentos o incluso la voluntad de imponer una determinada posición.

El documento desarrolla cada una de las 20 competencias de cultura democrática con descriptores que distinguen los niveles "básico", "intermedio" y "avanzado" (2018b). También concreta las implicaciones que tales competencias tienen en el desarrollo del currículum, la enseñanza, la evaluación, la formación del profesorado y la cultura o entorno escolar (2018c). Es importante destacar la inclusión de un capítulo específico sobre la prevención de la radicalización extremista y/o terrorista, donde se defiende que una educación articulada sobre las competencias de cultura democrática es clave para la prevención de la radicalización (2018c), en tanto que permite que las personas jóvenes (1) analicen de manera crítica las acciones y los discursos extremistas y puedan rechazarlos; (2) eviten las explicaciones simplistas que separan ellos de nosotros, base de las políticas fascistas (STANLEY, 2018); (3) entiendan y respeten las creencias y cosmogonías de otras personas; (4) comprendan que las herramientas democráticas no violentas son más efectivas para defender las opiniones y conseguir las causas políticas y sociales; y (5) valoren la dignidad humana y los derechos humanos. 
El énfasis en la prevención de la radicalización ha aumentado durante la última década, de acuerdo con los contextos y la historia reciente vivida en diferentes países europeos (DIALLO; EMBARKI; BEN ABDALLAH, 2016). Algunos ataques terroristas producidos durante los últimos años, ejecutados por ciudadanos nacidos y educados en los mismos países de producción de los atentados, dispararon las alertas sociales, políticas y educativas. Ha tenido impacto a nivel general europeo, y sobre todo en algunos países en particular. Este es el caso, por ejemplo, de Francia (DIALLO; EMBARKI; BEN ABDALLAH, 2016). Después de los atentados de 2015, el debate sobre la Educación para la Ciudadanía en Francia tomó gran protagonismo no sólo en el ámbito educativo, sino también político e institucional, junto a los debates sobre la crisis de integración (BROUARD; TIBERJ, 2008; DOMINIQUE, 2009; LABORDE, 2004), sobre marginalización, desigualdades económicas y estigmatización de grupos minoritarios (DIALLO; EMBARKI; BEN ABDALLAH, 2016).

La propuesta del Consejo de Europa opta por una aplicación de las competencias transdisciplinares, de manera que tienen que trabajarse de manera transversal en las diferentes asignaturas de todos los niveles educativos de educación obligatoria. Una mayor concreción a esta premisa se hace compleja, al tener los diferentes países estructuras curriculares y etapas formativas muy variadas entre sí. Aun así, la incorporación de una Educación para la Ciudadanía basada en el trabajo de las competencias de cultura democrática, no se inscribe sólo en el marco de las diferentes asignaturas, sino también en unas metodologías que favorezcan el diálogo y el aprendizaje cooperativo y deliberativo, y en una cultura escolar centrada en el compromiso de toda la comunidad educativa, que incentive la participación y la toma de decisiones conjunta en la escuela. En parte aboga por el tipo de escuela que Biesta (2006, p.136) proyecta cuando se pregunta: "what kind of schools do we need so that children can act?".

\section{LA INVESTIGACIÓN SOBRE EL ESTADO DE LA EDUCACIÓN PARA LA CIUDADANÍA EN EUROPA}

El Consejo de Europa no sólo se orienta al diseño de propuestas para la implementación de la Educación para la Ciudadanía, sino que también realiza estudios para conocer el estado de esta en los diferentes países y contextos. En 2004, el Consejo de Europa encargó un estudio para conocer la situación de la Educación para la Ciudadanía Democrática en las diferentes regiones europeas. En el trabajo (BÎRZÉA et al., 2004) se constatan algunas diferencias en los enfoques de los diferentes países, aunque comparten unos fundamentos similares: la educación para la democracia, la educación para la paz y la educación en derechos humanos. Los resultados del estudio indican que todos los países dan importancia a la construcción de la identidad nacional en los planteamientos de la Educación para la Ciudadanía, la construcción de una conciencia global y la necesidad de diálogo entre diferentes identidades. Los autores destacan que:

In spite of some differences in national approaches to policy development in the countries, the main principles of educational reform are similar throughout the region. They stress democratization, humanism, decentralization, autonomy, flexibility, accountability, personal development, national identity and global awareness. EDC is defined, and declared, a priority in the area of societal and educational development. (BîRZÉA et al., 2004, p.92).

En 2005, Eurydice realizó un estudio en el que se concluía que en la mayoría de currículos de los países europeos se incluye la dimensión europea, priorizando aspectos económicos y políticos. Algunos países como Noruega, Reino Unido, Suecia, los Países Bajos y la comunidad flamenca de Bélgica, incluyen una dimensión internacional con más presencia que la dimensión europea. En cambio, los otros países presentan unos currículos con poca proyección internacional, algo que desde el estudio se recomienda corregir.

En 2010 los estados miembros de la Unión Europea adoptaron la Carta del Consejo de Europa sobre la Educación para la Ciudadanía y los Derechos Humanos. En ese momento, Eurydice realizó una nueva investigación para conocer cómo habían evolucionado las políticas educativas en relación a la Educación para la Ciudadanía, el currículo, la cultura escolar, la formación del profesorado y la participación activa del alumnado. Dicha investigación muestra que muchos de los países ya incluyen la perspectiva internacional y multicultural en los currículos, y que se proyecta la idea de la ciudadanía global. Se reafirma la importancia de la participación del alumnado más allá del centro, y en el compromiso con la defensa de 
los derechos humanos en los diferentes espacios y ámbitos de su vida cotidiana. En 2017 el Consejo de Europa publicó un nuevo estudio sobre la situación de la Educación para la Ciudadanía Democrática y los Derechos Humanos, que concluía: "all countries have commited to ensuring that learners are provided with the knowledge and the skills to promote human rights and global citizenship" (COUNCIL OF EUROPE, 2017, p.11).

A pesar de los avances, uno de los problemas que se detectan en los estudios realizados es la falta de formación específica del profesorado (EURYDICE, 2005, 2012). Se señala que: "When looking at teacher education, the general picture reveals that more efforts are needed to strengthen teachers' competences in teaching citizenship". (EURYDICE, 2012, p.15). Se detecta que las oportunidades de una formación específica son poco frecuentes, y que solo se evidencian en países como Austria o el Reino Unido. En otros países se han desarrollado programas y materiales específicos, pero sin opción a formarse a través de cursos específicos de formación inicial o continuada (GOMES et al., 2020). El Consejo de Europa considera fundamental que los diferentes países formen al profesorado en competencias profesionales para la Educación para la Ciudadanía Democrática y los Derechos Humanos, en una clara voluntad de prevención de las radicalizaciones extremistas, a partir del desarrollo del pensamiento crítico y la participación en la cultura democrática. Defiende que:

Teachers need to receive training on how to educate children on such sensitive topics [violent extremism] and hace the skills to attend to the needs of individual students while managing conflict. Students need to be given space to ask questions and develop critical thinking while building respect for difference (COUNCIL OF EUROPE, 2017, p.34).

Desde el mismo consejo se entiende que la formación del profesorado es fundamental, porque sólo con esta puede llegar a implementarse una enseñanza en base a las competencias de cultura democrática: "The CDC [Competences for Democratic Culture] approach would remain theory without properly educated and trained teachers; teachers are the only ones in a position to implement it in the field" (COUNCIL OF EUROPE, 2018c, p.76). Para hacerlo, defienden una doble responsabilidad, primero la del profesorado, que para poder enseñar competencias de cultura democrática ha de tener habilidades, actitudes y prácticas democráticas, y segundo, la de las instituciones. Sobre estas se escribe:

Teacher education institutions are responsible for enhancing study programmes in which future teachers are educated and trained, and providing practising teachers with highquality in-service courses, newly developed teaching materials and aids, new teaching methods and so on. It is equally important to engage in research and innovation projects which provide bases for improving existing practice both in schools and within teacher education institutions themselves. (COUNCIL OF EUROPE, 2018c, p.77).

El cumplimiento de estos planteamientos en la formación del profesorado no es sencillo. Como se argumenta desde el mismo Consejo, la formación del profesorado es muy diferente en los diferentes países, e incluso en las diferentes regiones de un mismo estado. No se trata sólo de diferencias en el contenido de la formación, sino también de diferencias en la formación académica. Algunos países entienden que la formación del profesorado tiene que darse en forma de Máster, en otros se da como Grado; tampoco todos los perfiles docentes se entienden desde la misma lógica. A esta diversidad se le añade la complejidad de los contextos en los que se da la formación, ya que en muchos casos hay una combinación entre instituciones universitarias y no universitarias (COUNCIL OF EUROPE, 2018c). En este sentido, Gomes et al (2020) destacan que en muchos países en los que no hay un compromiso estatal claro hacia la ED, son asociaciones no gubernamentales las que ofrecen formación, materiales y recursos para el profesorado y para las escuelas, a fin de promover la educación para una ciudadanía global crítica.

Como señalan estos autores, formar al profesorado europeo en educación para la ciudadanía democrática y global no es sencillo, ya que requiere conocimientos históricos y actuales de los problemas y conflictos contemporáneos. Además, también debemos formar a los profesores en las dudas y las preguntas que surgen del análisis de la realidad social (GOMES et al., 2020). Al final, el profesorado y el alumnado viven en un mismo contexto y afrontan retos, dudas e incertidumbres comunes. La exposición a los relatos discriminatorios y discursos populistas, la presencia de los contextos digitales, la vivencia de 
las desigualdades, el desarrollo de la vida en un mundo globalizado o las consecuencias del cambio climático, son experiencias que compartimos todas las generaciones. Entender que los retos que planteamos para el alumnado también comprometen al profesorado, nos obliga a reflexionar sobre las competencias docentes y sobre qué, cómo y por qué enseñar, para formar en las competencias de cultura democrática.

\section{RETOS Y CONCLUSIONES}

Esta revisión nos permite ubicar las bases de la Educación para la Ciudadanía en Europa y entenderla en la compleja dicotomía entre las particularidades de cada país y los esfuerzos y directrices globales. Los diferentes países presentan tradiciones y culturas democráticas propias, y expresan en los currículos escolares la voluntad de desarrollar las identidades nacionales a través de éstos. Por otro lado, aspiran a visiones más globales e interculturales, visiones que han ido aumentando la presencia en los últimos años. El Consejo de Europa pretende defender los derechos humanos y se focaliza desde hace décadas en su implementación y en su evaluación, y de las Competencias de Cultura Democrática en los diferentes países. A través de trabajos de investigación e informes específicos es posible conocer la situación en las diferentes naciones europeas, para poder detectar las flaquezas y las posibilidades a la hora de diseñar programas concretos, para que sean asumibles por los diversos sistemas educativos y que respondan a los retos del presente y del futuro.

Los retos para la Educación para la Ciudadanía en Europa toman, a nuestro entender, una doble vía: los retos educativos y los retos de la ciudadanía -si es que hay diferencia alguna entre estas dos dimensiones. En primer lugar, entendemos que los retos educativos van en la dirección de asegurar que la experiencia educativa sea una experiencia democrática, a fin de consolidar y realmente aprehender las competencias de cultura democrática. Para Biesta (2006), la cuestión no está en cómo podemos enseñar los conceptos o los valores democráticos en las escuelas, sino si el alumnado puede vivir o ejercer la democracia en la escuela: "wheter children and students can actually be democratic persons in the school" (p.138). Este autor entiende la democracia como el compromiso hacia un mundo de pluralidad y diferencia, el compromiso hacia un mundo en el que pueda aflorar la libertad. Para ello, defiende unas escuelas en las que haya acción democrática y en las que todo el alumnado sea considerado plenamente parte de la ciudadanía.

Con los retos de la ciudadanía nos referimos al contexto con el que se ha introducido el artículo: un presente que proyecta un futuro incierto con enormes retos globales a los que hacer frente. Aunque muchos de estos retos son planetarios, Europa afronta algunos de ellos de manera más específica: el aumento de los grupos neonazis; las políticas de la muerte en el blindaje de las fronteras frente a las olas de personas refugiadas; el éxito de discursos populistas, muchos de ellos centrados en relatos discriminatorios; las crecientes desigualdades económicas en países tradicionalmente entendidos como "ricos", o las responsabilidades del modelo económico desarrollado frente al ecocidio y a la emergencia climática. También la disminución del interés en las estructuras y la participación democrática, la euroescepcia y el aumento de nacionalismos excluyentes, son retos que afronta la ciudadanía europea, más allá de los retos globales y planetarios que atañen a toda la humanidad. Retos complejos que es necesario entender y analizar de manera crítica y detenida, pero que podríamos sintetizar en la expresión de Levinas (1989), cuando afirma que el mayor reto que afrontamos desde la educación democrática en la formación de la ciudadanía es entender que "estar en el mundo es estar en el mundo con otras personas".

Europa como casa común tiene sentido si los valores democráticos que se enseñan en la escuela atienden a los retos anteriores y se transmite una idea de democracia basada en la libertad, la igualdad, la equidad, la solidaridad y la justicia social. Hemos de defender una idea de Educación para la Ciudadanía en Europa que anteponga estos valores, que han sido la esencia de la historia y de la tradición democrática europea. Con este fin, esta perspectiva educativa europea debe contemplar algunos principios esenciales para la convivencia y la prosperidad conjunta, y que se plantean a continuación:

1. La idea de democracia debe contemplarse como un camino a seguir, un ideal que nunca se alcanza del todo, que hay que proteger y mejorar cada día (SANTISTEBAN; PAGÈS, 2007). 
2. La idea de libertad debe enseñarse relacionada con el bien común, somos libres para participar y mejorar nuestro entorno (VEUGELERS, 2018).

3. La justicia social es la esencia de la democracia, así como la solidaridad, el compromiso y la responsabilidad social deben ser valores ineludibles de la ciudadanía europea (SANTI; LEWIS; DELGADO; ROSS, 2017).

4. La identidad de cada nación o cultura debe protegerse, pero debe ser la base de la comprensión y el respeto de otras identidades o culturas (SANTISTEBAN; GONZÁLEZ-MONFORT, 2019).

5. Europa debe entenderse como una región abierta al resto del mundo, con una mirada de educación para la ciudadanía global (DAVIES, et al., 2018).

6. Una educación democrática europea debe tener la vocación de hacer visibles a personas e identidades que ahora son invisibles en los estudios sociales (HERNÁNDEZ CARRETERO; GARCÍA RUÍZ; DE LA MONTAÑA, 2015).

7. Europa no puede presentarse como un espacio sin dificultades, los problemas y conflictos sociales internos deben ser tratados en la Educación para la Ciudadanía, como parte de su enseñanza (LEGARDEZ; SIMONNEAUX, 2006).

La Educación para la Ciudadanía debe promover el amor por la ciencia y por la verdad, a partir de la formación del pensamiento crítico, reflexionando sobre el papel de las emociones y de la racionalidad (MASSIP, CASTELLVÍ, GONZÁLEZ-VALENCIA, 2020).

Por último, queremos señalar que una Educación para la Ciudadanía en la Europa del siglo XXI debe tener en los Derechos Humanos el punto de encuentro de pueblos y culturas, a partir de los cuales se debe promover la lucha contra el fascismo, el racismo, el sexismo, la homofobia, la aporofobia (CORTINA, 2017) u otras formas de discriminación. Hemos de educar en competencias para deconstruir los discursos del odio (ARROYO et al., 2018) y para construir contrarelatos alternativos, basados en la convivencia y la paz en Europa y en el mundo.

\section{REFERENCIAS}

ARROYO, Aurora; BALLBÉ, Maria; CANALS, Roser; GARCÍA, Carmen Rosa; LLUSÀ, Joan; LÓPEZ, Manuel; OLLER, Montserrat; SANTISTEBAN, Antoni. El discurso del odio: una investigación en la formación inicial. In LÓPEZ TORRES, Esther; GARCÍA RUIZ, Carmen Rosa; SÁNCHEZ AGUSTÍ, María. Buscando formas de enseñar. Investigar para innovar en Didáctica de las Ciencias Sociales. Valladolid: Universidad de Valladolid y AUPDCS, 2018. p. 413-424.

BIESTA, Gert. Beyond Learning: Democratic Education for a Human Future. Routledge. Taylor and Fracis Group, 2006.

BîRZÉA, César; KERR, David; MIKKELSEN, Rolf; FROUMIN, Isak; LOSITO, Bruno; POL, Milan; SARDAR, Mitja. All-European Study for Democratic Citizenship. Strasbourg: Council of Europe, 2004.

BROUARD, Sylvain; TIBERJ, Vincent. France facing multicultural diversity: is l'intégration à la française really in crisis? In ARIANE, C y SIMON, R. Immigration, integration, and security: America and Europe in comparative perspective. Pittsburgh: University of Pittsburgh Press, 2008. P.1-22

CORTINA, Adela. Aporofobia, el rechazo al pobre. Un desafío para la democracia. Barcelona: Paidós, 2017.

COUNCIL OF EUROPE. Competences for Democratic Culture. Living together as equals in culturally diverse democratic societes. Council of Europe, 2016

COUNCIL OF EUROPE. Learning to live together. Council of Europe report on the state of citizenship and human rights education in Europe. Strasbourg: Council of Europe, 2017.

COUNCIL OF EUROPE. Reference Framework of Competences for Democratic Culture, 2018.

DAVIES, Ian; HO, Li Ching; KIWAN, Dina; PECK, Carla; PETERSON, Andrew, SANT, Edda; WAGHID, Yusef. 
Palgrave Handbook of Global Citizenship and Education. London: Palgrave Macmillan, 2018.

DIALLO, Ibrahima; EMBARKI, Mohamed; BEN ABDALLAH, Kaouthar. Education for citizenship at school in France: trajectory, tensions and contradictions. In PETERSON, Andrew; HATTAM, Robert; ZEMBYLAS, Michalinos y ARTHUR, James. The Palgrave International Handbook of Education for Citizenship and Social Justice. London: Palgrave Macmillan, 2016. p.391-409.

DUPREZ, Dominique. Urban rioting as an indicator of crisis in the integration model for ethnic minority youth in France. Journal of Ethnic and Migration Studies. v. 35, n. 5, 2009, p.753-770.

EURYDICE. Citizenship Education at School in Europe. Strasbourg: European Comission, 2005.

EURYDICE. Citizenship Education in Europe. Strasbourg: European Comission, 2012.

GALLAGHER, Tony; DUFFY, Gavin. Education for Citizenship Education and Social Justice in Northern Ireland. In PETERSON, Andrew; HATTAM, Robert; ZEMBYLAS, Michalinos y ARTHUR, James. The Palgrave International Handbook of Education for Citizenship and Social Justice. London: Palgrave Macmillan, 2016. p.523-544

GARCÉS, Marina. Ciutat princesa. Barcelona: Galàxia Guteberg, 2018.

GOMES, Alfredo; GÓMEZ, Ernesto; SANTISTEBAN, Antoni; PAGÈS, Joan. Europa. Global Citizenship Education and Teacher Education in Spain and Portugal. In Schugurensky, Daniel y Wolhuter Charl. Global Citizenship Education in Teacher Education: Theoretical and Practical Issues. London: Routledge, 2020. (en prensa)

HALLDÓRSDÓTTIR, Brynja; PÁLL JÓNSSON, Ólafur; RÓS MAGNÚSDÓTTIR, Berglind. Education for Democracy, Citizenship and Social Justice: The Case of Iceland. In PETERSON, Andrew; HATTAM, Robert; ZEMBYLAS, Michalinos; ARTHUR, James. The Palgrave International Handbook of Education for Citizenship and Social Justice. London: Palgrave Macmillan, 2016. p.435-463

HERNÁNDEZ CARRETERO, Ana María; GARCÍA RUIZ, Carmen Rosa; DE LA MONTAÑA, Juan Luis. Una enseñanza de las ciencias sociales para el futuro: recursos para trabajar la invisibilidad de personas, lugares y temáticas. Cáceres: Universidad de Extremadura/AUPDCS, 2015. p.383-393.

LABORDE, Cécile. Republican Citizenship and the Crisis of Integration in France. In BELLAMY, Richard ; CASTIGLIONE, Dario ; SANTORO, Emilio. Lineages of European Citizenship. London: Palgrave MacMillan, 2016. P.46-72.

LACLAU, Ernesto. The Populist Reason. London: Verso, 2005.

LEGARDEZ, Alain ; SIMONNEUAUX, Laurence. L'école à l'épreuve de l'actualité - Enseigner les questions vives. ESF, Issy-les-Moulineaux, 2006.

LEVINAS, Emanuel. Ethics as First Philosophy.In Hans, S. The Levinas Reader Oxford: Basil Blackwell, 1989. p. $75-87$.

MASSIP, Mariona; CASTELLVÍ, Jordi; GÓNZALEZ-VALENCIA, Gustavo. Entre la emoción y el pensamiento crítico: un estudio con profesorado de ciencias sociales en formación. In DÍEZ, Enrique Javier; RODRíGUEZ FERNÁNDEZ, Juan Ramón. Educación para el Bien Común. Hacia una práctica crítica, inclusiva y comprometida socialmente. Barcelona: Octaedro, 2020. p. 535-543

PICKETT, Kate; WILKINSON, Richard. The spirit level: why greater equality makes societies stronger. Nueva York: Bloomsury Press, 2009.

RANIERI, Maria. Populism, Media and Education. Challenging Discrimination in contemporary digital societies. Routledge Taylor \& Francis Group, 2016.

SANT, Edda. Democratic Education: A Theroretical Review (2006-2017). Review of Educational Research, $v$. 89, n. 5, 2019, p.655-696.

SANT, Edda; DAVIES, Ian; PASHBY, Karen; SHULTZ, Lynette. Global Citizenship Education. A Critical Introduction to Key Concepts and Debates. Chennai: Bloomsbury, 2018. 
SANT, Edda; LEWIS, Sue; DELGADO, Sandra; ROSS, Wayne. Justice and global citizenship education. In DAVIES, Ian; HO, Li Ching, KIWAN, Dina; PECK, Carla; PETERSON, Andrew; SANT, Edda; WAGHID, Yusef. Palgrave Handbook of Global Citizenship and Education. London: Palgrave Macmillan, 2018. p.227-243.

SANTISTEBAN, Antoni; GONZALÉZ-MONFORT, Neus. Education for Citizenship and Identities. In PINEDAALFONSO, José Ángel, DE ALBA-FERNÁNDEZ, Nicolás y NAVARRO-MEDINA, Elisa. Handbook of Research on Education for Participative Citizenship and Global Prosperity. Hershey PA, USA: IGI Global, 2019. p.551567

SANTISTEBAN, Antoni; PAGÈS, Joan. El marco teórico para el desarrollo conceptual de la Educación para la Ciudadanía. In PAGÈS, Joan; SANTISTEBAN, Antoni. Educación para la ciudadanía. Madrid: Wolters Kluwer, 2007.

STANLEY, Jason. How fascism works: the politics of us and them. London: Penguin Random House, 2018.

VEUGELERS, Wiel. La educación moral y para la ciudadanía en el siglo XXI. In MINGUEZ VALLEJOS, Ramón; ROMERO SANCHEZ, Eduardo. La educación ciudadana en un mundo en transformación: miradas y propuestas. Barcelona: Octaedro, 2018. p.121-140.

Recebido em: 29/03/2020

Revisões recebidas em: 21/04/2020

Aceito em: 21/04/2020

Publicado em: 14/07/2020 